\title{
Chapter
}

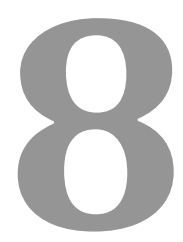

\section{INNOVATIVE NEXT-GENERATION PLGA MICROPARTICLES WITH MULTIFUNCTIONAL ARCHITECTURE}

\section{Eric Sah $^{1}$ and Hongkee Sah ${ }^{2 *}$}

${ }^{1}$ College of Science, University of Notre Dame, Notre Dame, IN 46556, USA

${ }^{2}$ College of Pharmacy, Ewha Womans University, 52 Ewhayeodae-gil, Seodaemun-gu, Seoul 03760, Republic of Korea 


\section{Contents}

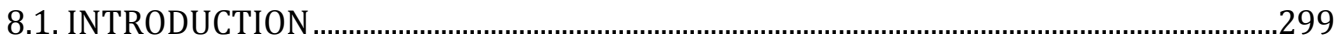

8.2. GOLF BALL-LIKE PLGA MICROSPHERES WITH DIMPLED SURFACE ...............................299

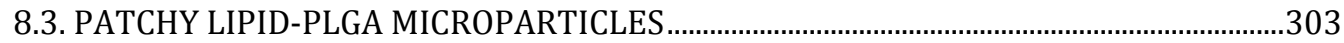

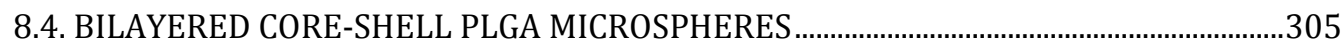

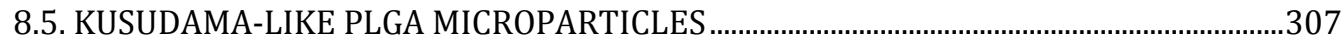

8.6. PLGA MICROPARTICLES WITH ANY GEOMETRICAL MICROFEATURE ..........................308

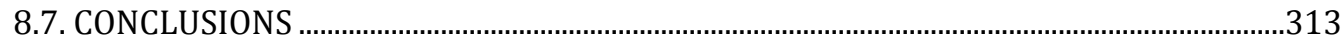

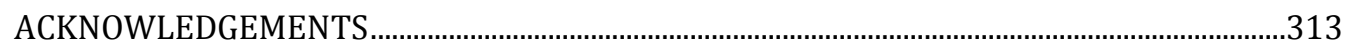

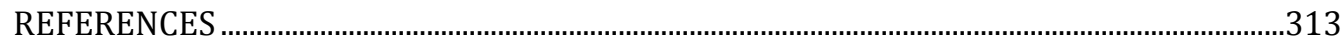




\subsection{INTRODUCTION}

Poly(lactide-co-glycolide) (PLGA) microspheres are used in a wide array of applications from drug delivery to cosmetic fillers. Commercial microsphere products include Arestin $^{\circledR}$, Bydureon $^{\circledR}$, Decapeptyl ${ }^{\circledR}$ SR, Lupron Depot ${ }^{\circledR}$, Risperdal Consta ${ }^{\circledR}$, Sandostatin ${ }^{\circledR}$ LAR Depot, Vivitrol ${ }^{\circledR}$, and Sculptra ${ }^{\circledR}$ Aesthetic. All these microsphere products have non-porous matrices. PLGA degradation rate plays a decisive role on the rate and kinetics of drug release. Most of those PLGA microsphere products are fabricated using phase separation techniques or emulsion-templated microencapsulation techniques. PLGA microspheres prepared using these techniques are spherical in shape and show relatively wide size distributions. They also have a morphology that is either symmetrically monolithic matrix or cavities-filled matrix. In the past decade, pharmaceutical/materials scientists have further explored the potentials of intelligent next-generation PLGA microparticles. To engineer multifunctional PLGA delivery systems, innovative microfabrication techniques have been suggested. These evolving technologies allow the fabrication of versatile PLGA microparticles with highly monodisperse sizes, any desired geometry, and anisotropic features that are inconceivable by previous microparticle manufacturing processes. These tailor-made microparticles are advantageous in precisely controlling their characteristics and in vivo performance such as PLGA degradation, co-delivery of multi-drugs, co-encapsulation of dual drugs with totally different physical properties, drug release behavior, and cell interaction. This chapter introduces various kinds of PLGA microparticles that have peculiar morphology and set themselves apart from commercially available PLGA microsphere products.

\subsection{GOLF BALL-LIKE PLGA MICROSPHERES WITH DIMPLED SURFACE}

Golf ball-shaped microspheres can be created through various methods including seed dispersion, emulsion polymerization, pickering emulsion with small colloidal particles as emulsifiers, oil/water $(\mathrm{o} / \mathrm{w})$ emulsion using organic phase change materials (PCM), water/oil/water (w/o/w) emulsion using formulation approaches, or droplet imprinting [1-4]. A seed dispersion technique cannot be applied toward a pre-made polymeric material. In the practice of a pickering emulsion process, removal of solid colloidal particles used to stabilize emulsion leads to the formation of dimpled surface on the hardened microspheres. However, this pickering emulsion technique cannot control internal porosity of microspheres. On the other hand, either $\mathrm{o} / \mathrm{w}$ emulsion using PCM or w/o/w emulsion using formulation strategy can be used to make dimpled PLGA microspheres with the desired magnitude of 
internal porosity. Kimetal. introduced an $\mathrm{o} / \mathrm{w}$ emulsion using 2-methylpentane to make dimpled PLGA microspheres [5]. 2-Methylpentane, which is soluble in methylene chloride, cannot dissolve PLGA. In addition, 2-methylpentane can be removed easily during solvent evaporation process since it has a boiling point of $62^{\circ} \mathrm{C}$. On the basis of these physical properties, the authors used 2-methylpentane as PCM. By dissolving PLGA in methylene chloride containing 4\% 2-methylpentane and emulsifying this dispersed phase in water, they prepared an $\mathrm{o} / \mathrm{w}$ emulsion. Through solvent evaporation process, emulsion droplets were hardened into microspheres. The number and size of dimples as well as porosity inside the microspheres could be controlled by changing the weight ratio of PLGA:2-methylpentane. In an optimal condition, 2-methylpentane liquid bubbles form on the surface of emulsion droplets, and methylene chloride gets removed continuously by evaporation. As a result, PLGA and 2-methylpentane separate from one another. Based on the amount of 2-methylpentane used, the PCM can disperse evenly on microsphere matrix or form domains. Constant stirring removes 2-methylpentane colloidal bubbles on the surface and forms dimples. Pores also form because tiny 2-methylpentane residues on the microsphere matrix are removed. Figure 1 summarizes the mechanism for forming golf ball-like PLGA microspheres.

Schaefer and Singh used different PCMs (e.g., tricaprin, glycerol tricaprate) to prepare dimpled PLGA microspheres containing etoposide through a single $\mathrm{o} / \mathrm{w}$ emulsion method [6]. A dispersed phase was prepared by dissolving PLGA, tricaprin, and etoposide in methylene chloride, which was emulsified in an aqueous phase. The emulsion was subject to solvent evaporation to produce etoposide-containing PLGA microspheres. At first, the authors intended to increase the drug release rate by use of tricaprin. Actually, it did not affect etoposide encapsulation efficiency, but had impact on microsphere size and drug release rate. Absence of tricaprin led to the formation of PLGA microspheres with smooth matrix. However, including tricaprin into microsphere formulation resulted in the formation of a dimpled surface (Figure 2). Such results are consistent with those presented previously [7]. The authors postulated that dimples formed due to leaching of tricaprin into aqueous phase from emulsion droplets during solvent evaporation. However, considering that tricaprin is practically insoluble in water, more meticulous observations are needed to find out the pathway for how tricaprin is removed from emulsion droplets or embryonic microspheres. 


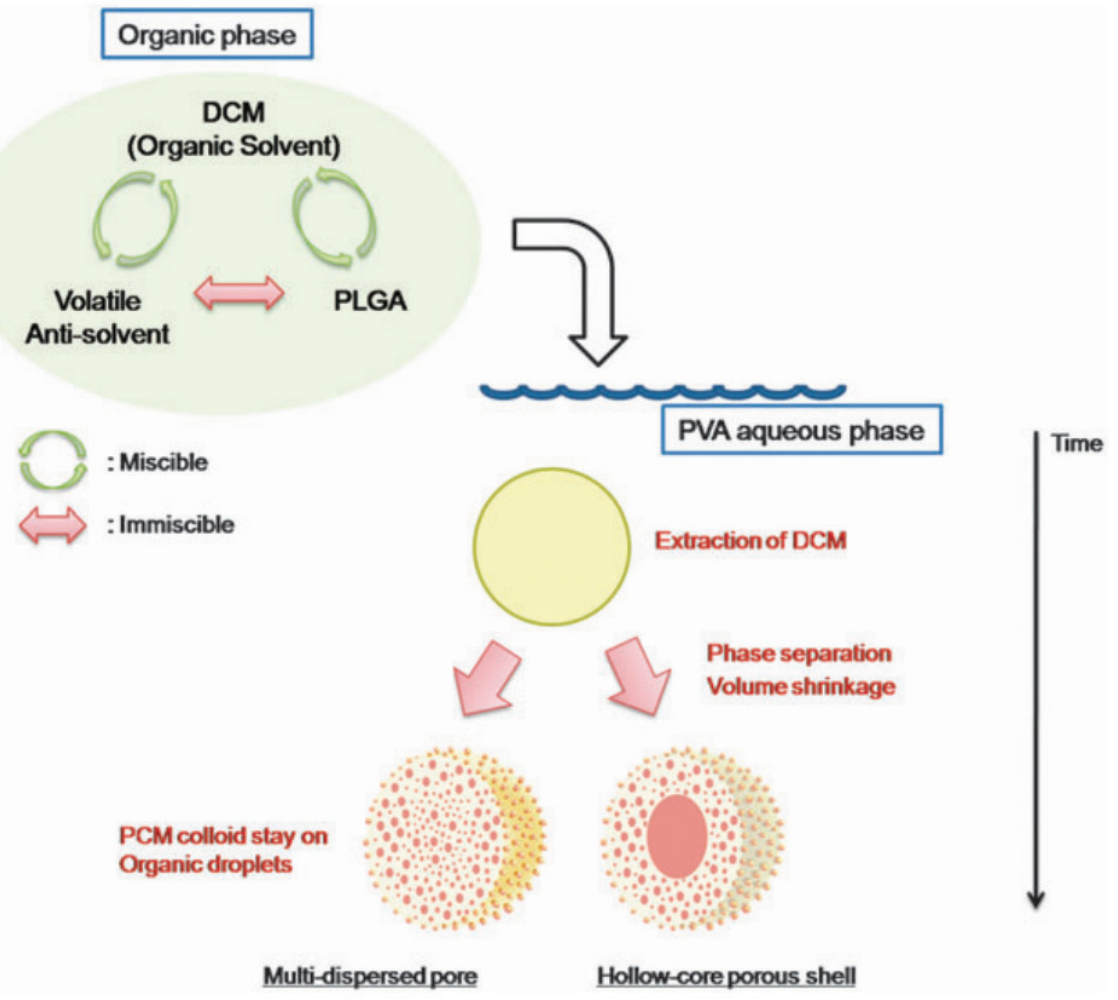

Figure 1. Diagram shows the mechanism of the formation of golf ball-like PLGA microspheres by an o/w emulsion method using a PCM. Dimpled PLGA microspheres with either hollow-core porous shell or multiporous matrix can be created by changing the weight ratio of PLGA:2-methylpentane (Reproduced from Ref. 5 with permission from The Royal Society of Chemistry).

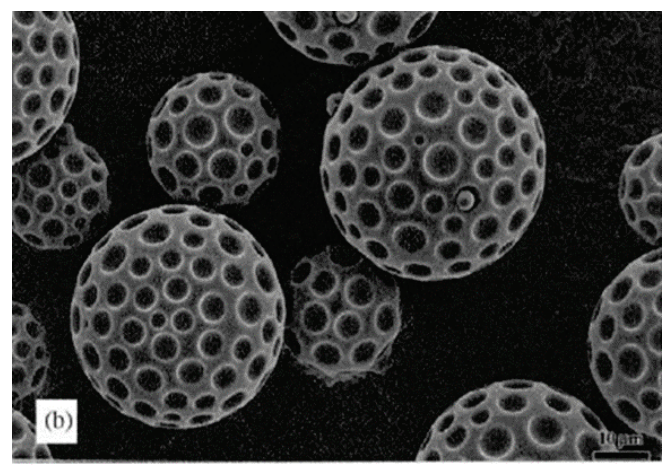

Figure 2. SEM micrograph of golf ball-like PLGA microspheres with dimpled surface (Reprined with permission from M.J. Schaefer and J. Singh. Biomaterials 23 (2002) 3465-3471. Copyright 2002 Elsevier Science Ltd.). 
The w/o/w double emulsion method can also be used to fabricate PLGA microspheres with dimpled surface. For instance, Mohamed and Walle followed w1/o/w2 double emulsion solvent evaporation to create golf ball-like PLGA microspheres containing plasmid DNA [8]. PLGA microspheres were formed when Pluronic L92 was dissolved in w1 that was used to make a primary emulsion. Methylene chloride was used as a dispersed solvent for PLGA. Interestingly, dimple morphology and characteristics of the microspheres were affected by the molecular weight of poly(propylene oxide) block. However, replacing Pluronic L92 with Tween in w1 led to the fabrication of dimple-free PLGA microspheres with smooth surface. Tween was able to stabilize the primary emulsion, but Pluronics acted as a poor stabilizer. Based on these results, the authors concluded that primary emulsion's instability is associated with the formation of PLGA microspheres with dimpled structure.

Lastly, the droplet imprinting method is also employed to produce dimpled PLGA microspheres [9]. In the droplet imprinting method, a high-shear mixer is used to disperse 2-methylpentane into an aqueous poly(vinyl alcohol) (PVA) solution. Separately, an o/w emulsion is prepared by emulsifying a dispersed phase consisting of PLGA and methylene chloride with PLGA in an aqueous phase. The aqueous 2-methylpentane dispersion and the o/w emulsion are combined together for use in the process of solvent removal. During the microsphere hardening process, 2-methylpentane colloidal droplets move on the surface of the emulsion droplets surface and then bounce back to the aqueous phase. This event leaves emulsion droplets imprinted with 2-methylpentane colloidal droplets. In other words, dimpled PLGA microspheres form as the surface of the emulsion droplets deforms due to 2methylpentane colloidal droplets and methylene chloride is removed by evaporation. The authors used this method to fabricate dimpled PLGA microspheres that are less than $10 \mu \mathrm{m}$. So far, 2-methylpentane has not been used as an ingredient for FDA-approved microsphere products. Thus, there would be concerns about residual 2-methylpentane in microspheres. It would be more appropriate to use ingredients that have been used in FDA-approved pharmaceutical products to create dimpled PLGA microspheres.

Golf ball is constructed to have a dimpled surface structure that can lengthen the distance of flight by reducing air resistance. Dimpled polymeric microspheres having a similar structure would experience less resistance toward fluid flow and decrease the drag force by air. Therefore, dimpled and hollow PLGA microspheres with appropriate size and density would be particularly advantageous for pulmonary drug delivery and drug deposition in the lung. 


\subsection{PATCHY LIPID-PLGA MICROPARTICLES}

Patchy microparticles are categorized as micron-sized particles that are anisotropically patterned by altering either surface chemistry or shape. Lipid-polymeric microparticles, which are one type of patchy microparticles, can be made of a lipidic material and PLGA. For example, Rasheed etal. used the strong van der Waals interaction between lipid-PEGylated functional groups (LPFGs) and PLGA to create lipid-polymeric patchy type particles [10]. Figure 3 is a scanning electron microscopic image that shows the exterior and interior of a single patchy LPFG-PLGA microparticle.

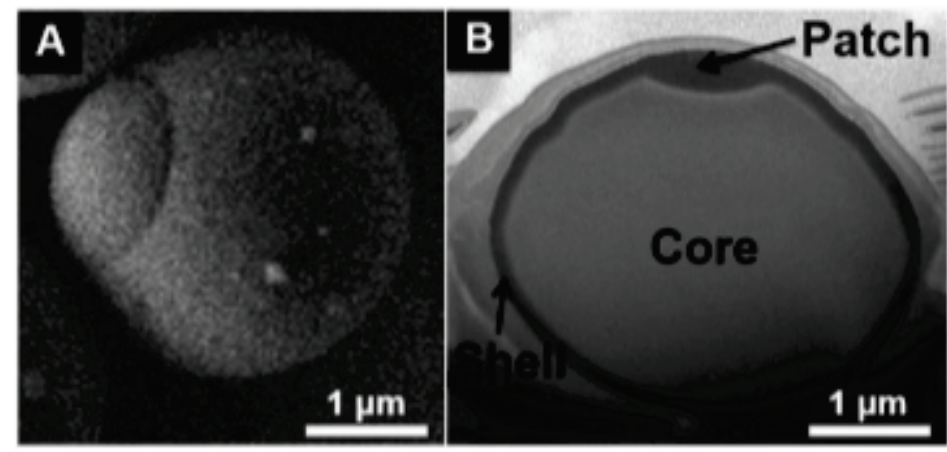

Figure 3. The external and internal morphology of a patchy microparticle with a patch-shell-core structure. Prior to the focused ion beam cross-section, the microparticle was coated with gold-palladium alloy to prevent damage by the ion beam (Reprinted with permission from N. Rasheed et al. Langmuir 31 (2015) 6639-6648. Copyright 2015 American Chemical Society).

Examples of LPFGs include 1,2-distearoyl-sn-glycero-3-phosphoethanolamine-poly(ethylene glycol) (DSPE)-PEG with amino, methoxyl, or maleimide terminal groups. PLGA is used as a biocompatible, biodegradable polymer that functions as a hydrophobic core and can accommodate various ingredients. LPFGs can form not only a shell surrounding the hydrophobic PLGA core but also a single or multiple patches on the PLGA surface. LPFGs have been long used as FDA-approved ingredients extensively in drug delivery systems like liposome, polymeric nanoparticles, and lipid nanoparticles. LPFGs also are actively used as lipidic materials for the production of core-shell lipid-polymer hybrid nanoparticles. Core-shell lipid-polymer hybrid nanoparticles can be made by either a two-step method or a single-step method. A single step method based on solvent extraction/evaporation can be summarized as follows [11]. PLGA is dissolved in a water-immiscible solvent such as methylene chloride or ethyl acetate. LPFGs are dispersed in water containing ethanol with the aid of sonication, mechanical stirring, or homogenization. The polymeric solution is emulsified in 
the aqueous phase by homogenization or sonication. Solvent removal by evaporation and/or extraction transforms nanodroplets into polymeric nanoparticles coated with LPFGs.

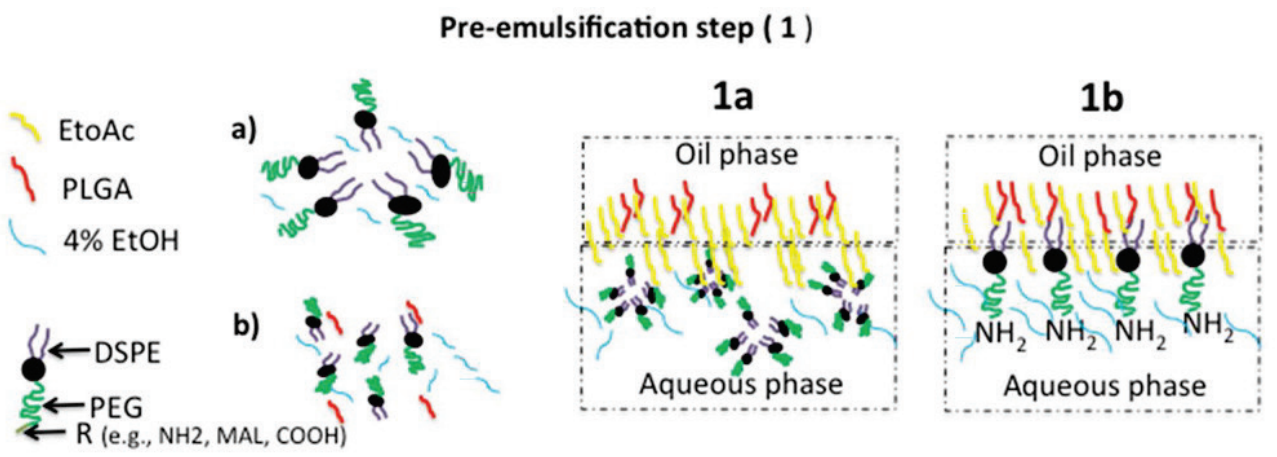

Emulsification step ( 2 )

Shear stress

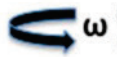

$\omega$

(a)

Shell formation

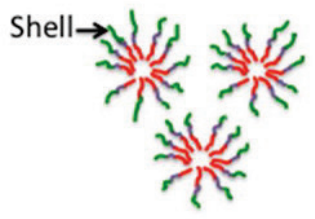

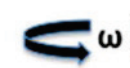

$\omega$

Patch formation

(b)

(c)

Droplets with a core-shell structure and patch

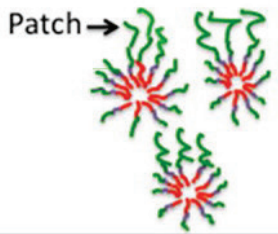

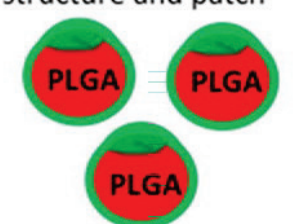

Figure 4. Schematic pathway to fabricate patchy type lipid-PLGA microparticles via a single-step solvent evaporation/extraction technique. In the step (1), PLGA and LPFGs interact with PLGA via hydrophobic interactions. Core-shell type microdroplets are formed during the emulsification step (2a), and a jammed pile resulting from the accumulation of LPFGs leads to the formation of a patch (2b). As solvent removal proceeds, the patchy core-shell microdroplets (2c) become patchy type microparticles.

EtoAc represents ethyl acetate, while EtOH stands for ethanol (Reprinted with permission from N. Rasheed et al. Langmuir 31 (2015) 6639-6648. Copyright 2015 American Chemical Society).

Rasheed etal. modified the above single-step solvent evaporation and extraction method to produce patchy PLGA microparticles [10]. Emulsifying a polymeric organic solution in an aqueous lipidic material-containing dispersion by high-energy or high-shear mixing devices usually leads to the formation of nanodroplets. However, the authors used relatively low shear stress to make microdroplets instead of nanodroplets. During the fabrication process, the DSPE fragment of the LPFGs interacts with PLGA via hydrophobic interactions, thereby leading to formation of the particle's shell. A jammed pile 
resulting from the accumulation of LPFGs appears as a patch. Figure 4 illustrates the entire pathway and mechanism of the fabrication of patchy PLGA microparticles. Among various process parameters, the shear stress applied for emulsification was identified as the most critical parameter that determined the number of patch (single vs. multiple) on the surface of the PLGA microparticles as well as their internal morphology (hollow vs. solid core) [12]. By controlling the shear stress, they were able to precisely control the patch-core-shell features. In particular, the authors showed how patchy particles were able to emit a natural photoacoustic signal. As those LPFGs contain amine or maleimide functional groups, they could be conjugated to a variety of ligands with desired functionality. Practically, the authors were able to improve photoacoustic signal by functionalization of patches with gold nanorods. Thus, those functionalized multipatchy particles can be utilized in biomedical imaging as photoacoustic contrast probes. Using different functional ligands, it might also be able to deliver these patchy type lipid-PLGA core particles to targeted tissues or organs.

\subsection{BILAYERED CORE-SHELL PLGA MICROSPHERES}

Electrospray, which is also known as electrohydrodynamic atomization, is a promising technique that can prepare electrospun fibers, homogeneous nanoparticles, and microparticles. Polymeric particles with typical matrix morphology are produced in a single axial electrospray process. The coaxial electrospray (CES) process, which was first introduced by Loscertalesetal., provides better flexibility in preparing multifunctional polymeric particles [13]. The CES process has been found to be effective not only in the encapsulation of various drugs with different physicochemical properties but also in the manipulation of microsphere morphology. For example, water-soluble macromolecules such as proteins, enzymes, and antibiotics are encapsulated by coaxial electrospraying without losing their integrity [14-16].

Yuan et al. reported a CES system consisting of a droplet generation module, a droplet collection module, and a process monitoring module [17]. The droplet generation module included a coaxial needle made of an inner needle and an outer needle. A beaker containing an aqueous PVA solution subject to stirring served as the droplet collection module. The outer liquid was an ethyl acetate solution in which $5-10 \%$ PLGA $(\mathrm{Mw}=10,000)$ was dissolved, which was responsible for forming the shell of their core-shell microspheres. A 2-5\% curcumin acetone solution containing $0.5-2 \%$ PLGA $(M w=50,000)$ was used as an inner solution to form a core matrix of the microspheres. By optimizing CES process parameters (e.g., applied electric voltage, and the flow rates of their inner and outer solutions), they were able to generate stable droplets from the coaxial cone. Since the microdroplets contained the organic solvents used to dissolve PLGA and curcumin, they were prone to massive aggregation 
when collected directly by a hard plate such as an aluminum foil. To avoid this limitation, the microdroplets were collected in the aqueous PVA solution being stirred and hardened into the solidified PLGA microspheres by the solvent removal process. At the end, they were successful in the fabrication of core-shell structured PLGA microspheres in which curcumin was loaded in the core. The analyses of confocal laser scanning microscopy and scanning electron microscopy proved the bilayered core-shell morphology. The bilayered PLGA microspheres had the mean diameter of $2.3 \mu \mathrm{m}$, and $48.8 \%$ of curcumin was loaded into the core-shell microspheres at encapsulation efficiency of $86.7 \%$. The in vitro drug release profile observed with the core-shell PLGA microspheres was demonstrated to be better than that attained with the conventional matrix-type PLGA microspheres.

Following coaxial electrospraying, Nie et al. produced core-shell microspheres loaded with multidrugs (i.e., paclitaxel and suramine) to achieve their sequential and parallel release [18]. To prevent the premature release of a drug in the core matrix of the core-shell microspheres, a slower degrading PLGA (poly-L-lactide with Mw of 85,000-160,000) was chosen to make the core-forming material. Based on this rationale, a faster degrading PLGA with a lactide : glycolide ratio of 50:50 and $\mathrm{Mw}$ of 40,000-75,000 was selected as the shell-forming material. In the practice of their CES process, the inner liquid consisted of the slower degrading PLGA, paclitaxel, and methylene chloride. On the other hand, the outer liquid was made of suramine sodium salt, ethanol, the faster degrading PLGA, and ethyl acetate. In order to avoid microdroplet fusion and microsphere aggregation, the microdroplets generated from their coaxial cone were collected with anhydrous ethanol rather than aluminum foil. This CES process provided the microencapsulation of two drugs with completely different hydrophobicity/hydrophilicity. Control of process parameters (e.g., the flow rate of the outer liquid and that of the inner liquid, applied voltage, polymer composition and concentration) leads to the fabrication of PLGA microspheres with different core-shell ratios. This in turn allows the manipulation that releases kinetics of multidrugs from the core-shell type microspheres. Zamani etal. also prepared protein encapsulated core-shell structured PLGA microspheres by CES [19]. A PLGA-dissolved solvent mixture of methylene chloride and dimethylformamide was employed as an outer liquid (shell), whereas an aqueous bovine serum albumin (BSA) solution was employed as an inner liquid (core). They classified the resultant microspheres as core-shell structured microspheres. Strictly speaking, they belong to typical hollow microspheres since the dried microspheres have hollow cavities encased by a PLGA shell. Thus, such a core-shell structured microsphere with a hollow cavity is beyond the scope of this chapter and not discussed here. 


\subsection{KUSUDAMA-LIKE PLGA MICROPARTICLES}

There are reports on a micron-sized particle displaying a porous sponge-like ball structure that resembles the decorative Japanese paper spheres known as "Kusudama" [20,21]. For example, following a new solid-in-oil-in-water (s/o/w) emulsion technique, Takai etal. prepared the Kusudama-type porous PLGA microspheres in which hydroxyapatite nanoparticles were dispersed [21]. In their microencapsulation process, PLGA was first dissolved in methylene chloride. Hydroxyapatite granules in the size of approximately $20 \mu \mathrm{m}$ were dispersed in the polymeric dispersed phase. This s/o dispersion was emulsified in an aqueous PVA solution by magnetic stirring. The resultant s/o/w emulsion was diluted in a large amount of an aqueous PVA solution containing $0.5 \mathrm{M} \mathrm{NaCl}$. The microspheres were hardened by solvent evaporation/extraction. The Kusudama-like morphology of PLGA microspheres, as seen in Figure 5, was attained only when both hydroxyapatite and $\mathrm{NaCl}$ were used in the microencapsulation process. The microspheres consist of two compartments of (a) a dense core and (b) a porous layer with an assembly of cone-like pores. In a broad sense, this microsphere type can be classified as a core-shell structured microsphere, but the layer of the microspheres has a unique cone-like porous skeleton that is similar to that of Kusudama. The mechanism of pore formation was ascribed to fragmentation of hydroxyapatite granules into nanoparticles, interaction between hydroxyapatite and PLGA, differences in solvent migration rate between core and surface layer during microsphere hardening, and a pickering emulsion effect of hydroxyapatite nanoparticles toward $\mathrm{NaCl}$-induced emulsion instabilization.

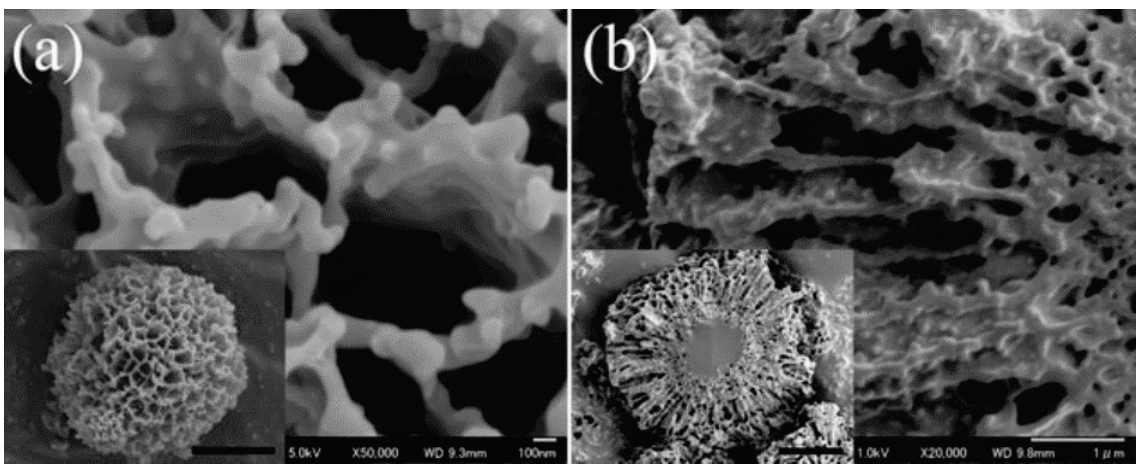

Figure 5. SEM micrographs of (a) surface and (b) cross-sectioned morphology of porous Kusudama-like PLGA microspheres. Scale bars in small magnifications are

$5 \mu \mathrm{m}$, whereas scale bars in high magnifications are (a) $100 \mathrm{~nm}$ and (b) $1 \mu \mathrm{m}$. (Reproduced from Ref. 21 with permission from The Royal Society of Chemistry). 
The deep, large pores of the Kusudama-like PLGA microspheres might be advantageous in loading biomacromolecules via adsorption or physical entrapment. They may also find applications in encapsulating hydrophobic drugs in their core and delivering hydroxyapatite to the bone tissue.

\subsection{PLGA MICROPARTICLES WITH ANY GEOMETRICAL MICROFEATURE}

Traditional methods to fabricate polymeric particles are usually based on emulsion-templated solvent evaporation/extraction techniques. However, their production yields can be low, and the resultant polymeric particles tend to exhibit heterogeneous populations as they have broad size distribution. Furthermore, it may be difficult to achieve high drug payloads in these polymeric particles. In order to avoid these limitations, soft lithographic techniques have been increasingly utilized for the preparation of shape- and size-specific smart nanoparticles that deliver drugs and/or imaging agents to targeted cells, tissues, or organs [22,23]. Those techniques can also be applied for the preparation of PLGA microparticles. The geometry of a lithographic replication mold can be designed to produce PLGA microparticles with any desired geometry such as trapezoidal-, conical-, bar-, arrow-, square-, rectangular-, or cross-shaped particles [24]. The so-called hydrogel template method proposed by Acharya etal. is an example of imprint lithographic replication techniques [25,26]. The hydrogel template method can be explained through the process mapping shown in Figure 6. First, a silicon wafer master template with a series of vertical posts is created (Figure 6A). A warm aqueous gelatin solution is poured on top of it (Figure 6B). After gelatin is solidified upon cooling, the gelatin mold is placed where cavities would be visible on top (Figure 6C). A drug-dissolved polymeric organic solution is poured onto the cavities (Figure 6D). After removing the organic solvent and dissolving the gelatin mold in water, polymeric microparticles can be collected through centrifugation or filtration.

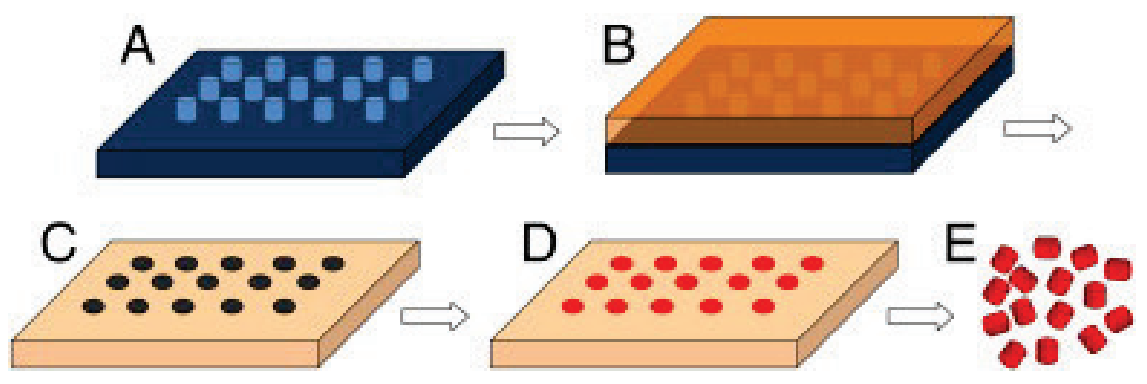

Figure 6. Schematic pathway of fabricating monodisperse microparticles using the hydrogel template technique (Reprinted with permission from G. Acharya et al., J. Control. Release 141 (2010) 314-319. Copyright 2009 Elsevier B.V.). 
In the original hydrogel template technique, gelatin was used as a moldforming material based on the following reasons: (a) gelatin has enough mechanical strength to form a template at an appropriate concentration, and (b) it has a gel-to-sol transition temperature that would not affect the stability of most drugs. However, storage of a gelatin template for an extended period of time can lead to microorganism contamination, and issues may arise in the physical integrity of the gelatin template. PVA template has been proposed to avoid these problems [27]. The authors first designed a silicon wafer master template containing cylinder wells of $10 \mu \mathrm{m}$ in diameter and $10 \mu \mathrm{m}$ in height. After coating and curing the master template with polydimethylsiloxane (PDMS), the resultant PDMS template was peeled off from the silicon wafer master template. The PDMS template was evenly coated by a $4 \%$ PVA solution consisting of water and ethanol. After completion of solvent evaporation, the appearing PVA template was detached from the PDMS template and was used as a mold for fabrication of microparticles. PLGA and drug (risperidone, methylprednisolone acetate, or paclitaxel) were dissolved in either benzyl alcohol-ethyl acetate cosolvent or methylene chloride. For each PVA template well, $60 \mu \mathrm{l}$ of the drug-containing polymeric organic solution was deposited. After solvent removal, the PVA template containing the hardened PLGA microparticles was dissolved in water. The microparticles were collected through centrifugation. This hydrogel template method produced drug-containing PLGA microparticles having target size less than $10 \mu \mathrm{m}$ (Figure 7). Also, the microparticle populations showed low variance in size distribution. Critical process parameters were found to be the type of organic solvent, PLGA properties (e.g., intrinsic viscosity), PLGA concentration, and the rate of solvent removal. Variations in these parameters led to the formation of different microparticle morphology such as cylindrical particles, irregularly shaped particles, and cup-like particles with hollow cores. Depending on drug type, encapsulation efficiency was within $82-92 \%$, and different drug release profiles were obtained with those microparticles. In a separate study, Malavia etal. loaded OHR1031, which was being developed as a treatment for glaucoma, onto PLGA microparticles by using the hydrogel template technology [28]. Their microparticles had the median size of $60 \mu \mathrm{m}$, and the drug encapsulation efficiency reached $100 \%$ at the drug payload of $57 \%$. Its release pattern followed a zero-order kinetics, and the drug release rate was constant for more than 3 months. PLGA microparticles with those characteristics have advantage as they can be put in ocular spaces to provide a long acting depot effect. The hydrogel template method appears to be promising because it allows flexibility in preparing homogeneous microparticles with desirable size and geometry. It also enables the encapsulation of higher amounts of various drugs into microparticles following a simple and facile process. Changing formulation or process parameters may help fabricate microparticles with target drug release profiles. 

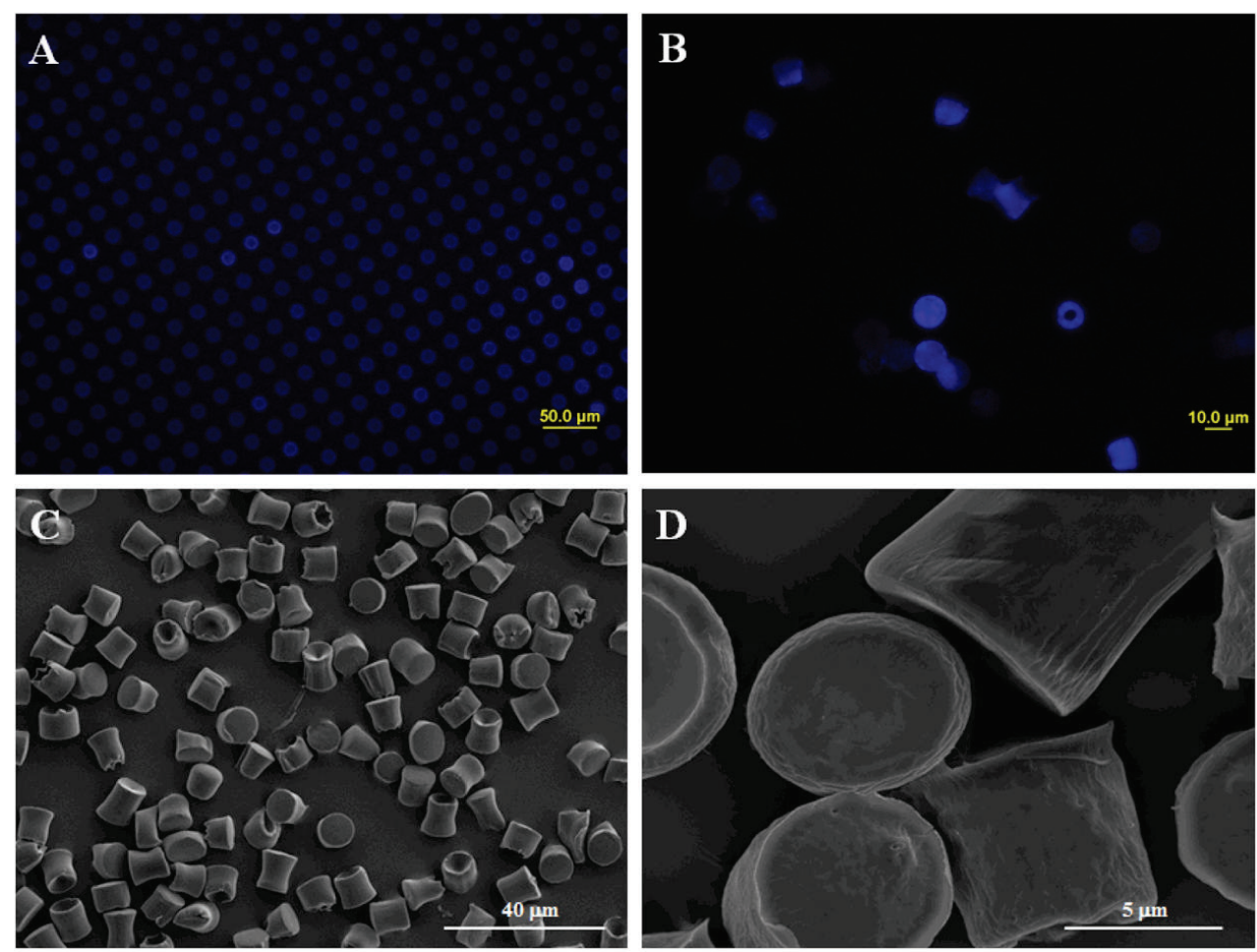

Figure 7. Fluorescent images of PLGA microparticles observed before (A) and after (B) dissolving PVA templates. SEM micrographs of PLGA microspheres collected after dissolving PVA templates (C and D) (Reprinted with permission from Y. Lu etal., Int. J. Pharm. 461 (2014) 258-269. Copyright 2013 Elsevier B.V.).

In general, traditional imprint lithography techniques face several limitations. For example, they usually produce scum layers/embossed films because the material to be molded has considerable affinity toward the surface of a mold template. In contrast to the conventional imprint lithography techniques, Rollandetal. developed the so-called Particle Replication In Nonwetting Templates (PRINT) technique that could produce scum-free, isolated nanoparticles or microparticles [29]. Instead of the commonly used PDMS, they used a perfluorinated polyether (PFPE) mold with highly fluorinated surface. It displayed a nonwetting property toward many organic materials. For example, a PFPE based device permits the flow of organic solvents such as methylene chloride, whereas a commonly used PDMS based one does not. This special feature enables the production of individual, discrete particles with various size, shape, and composition. They demonstrated that their PFPE mold has superior replication properties for use in lithographic applications. Also, this technique made it possible to fabricate monodisperse, shape-specific particles with various polymers such as poly(ethylene glycol diacrylate), triacrylate resin, poly(lactic acid), and poly(pyrrole). In the following work, Enlow etal. 
utilized the PRINT technique to encapsulate diverse bioactive agents such as proteins, DNA, and small-molecule therapeutics into these particles [30]. Figure 8 shows the process mapping of the PRINT technique used to prepare PLGA microparticles containing high payloads of docetaxel. If briefly explained, PLGA and docetaxel were dissolved in a mixture of dimethyl sulfoxide-dimethylformamide $(1: 4)$. This solution was casted on a sheet of polyethylene terephthalate (PET). Heat was applied to remove the organic solvents, and the appearing film was then placed in contact with the patterned side of the mold fabricated from a perfluorinated polyether elastomer. PLGA and docetaxel flowed into the cavities as they passed through a heated nip. When the heat was cooled, they solidified. The filled mold was then placed on a fresh sheet of PET, which was passed through a laminator without splitting to remove the particles from the mold. The mold was peeled from the PET, leaving an array of particles on the surface. Figure 8 also shows SEM micrographs of PLGA microparticles fabricated by the PRINT technique.

The shape of each particle is decided by the shape of the cavity in the elastomeric mold. Therefore, this technique can give precise control over the size and shape of each particle. It has been shown that highly monodisperse PLGA nano- and microparticles with shapes of cylinder, spheres, cubes with ridges, and particles have center holes [23]. Both hydrophilic and hydrophobic drugs can be easily encapsulated into these particles at high drug payloads. Also, their diverse sizes and shapes could be manipulated to investigate their effects upon cell uptake, in vivo distribution, and flow characteristics.

It is known that the size and charge of particles affect cellular internalization, systemic circulation, blood residence time, and in vivo distribution and deposition. Recent experimental results reveal particle geometry's (e.g., shape) impact on in vivo behavior as well [31-33]. It was also reported elsewhere that nonspherical particles show higher targeting efficiency than spherical particles and that elongated particles show more resistance compared to phagocytosis $[34,35]$. Decuzzi etal. stated that compared to spherical particles, "discoidal particles" travel laterally toward the blood vessel wall and can exhibit stronger affinity toward the blood vessel wall [36]. Most similar experiments tended to use nanoparticles. However, more experiments now involve the geometry of microparticles and how they influence the in vivo behavior. For example, the cellular internalization of cubic PEG hydrogel particles was compared with that of cylindrical PEG hydrogel particles by using HeLa cells $[37,38]$. HeLa cells were capable of uptaking up to $3 \mu \mathrm{m}$ cubic and cylindrical particles. However, $1 \mu \mathrm{m}$-sized cylindrical particles with 1 aspect ratio were subject to much higher cellular internalization rates compared to $2 \mu \mathrm{m}$ or $3 \mu \mathrm{m}$ cubic particles. As far as cylindrical particles are concerned, their internalization rate into HeLa cells was greatly influenced by their aspect ratio. Similarly, endocytosis or intracellular distribution of endothelial cells of PLGA particles impact particle geometry [39]. The PRINT technology can precisely control particle 
geometry and surface chemistry. It thus would give an opportunity to investigate more systematically their effects upon in vivo behavior of particles.

A)

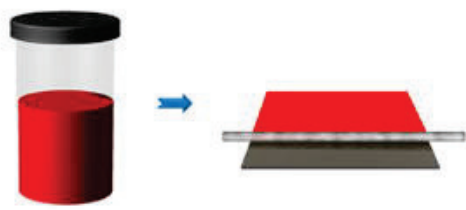

B)

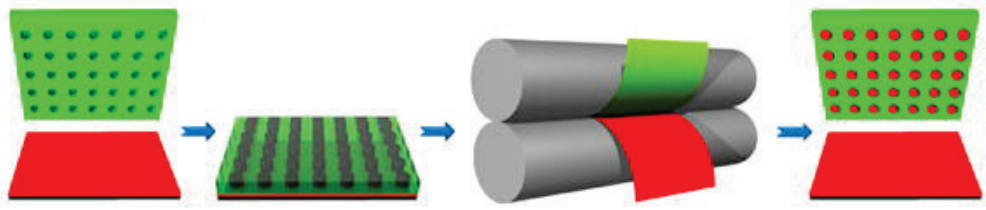

C)
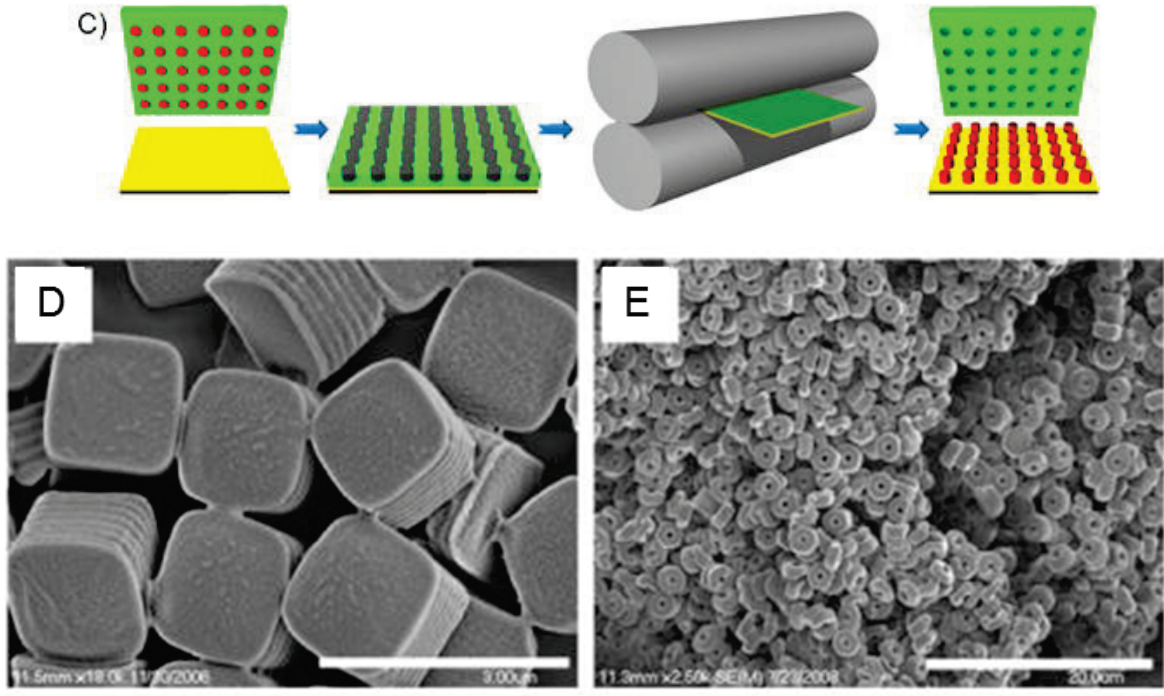

Figure 8. The process mapping of the PRINT technique and the morphology of PRINT fabricated PLGA microparticles. (A) Delivery Sheet Casting: PLGA and docetaxel are dissolved in a mixture of dimethyl sulfoxide and dimethylformamide (red). A mayer rod is then used to draw a film from this solution on a PET substrate. The solvent is removed under heat generating a solid state solution film referred to as the delivery sheet, as it will deliver the composition to the mold. (B) Particle fabrication: an elastomeric mold (green) in brought into contact with a PLGA (red) film, passed through a heated nip (gray) and split. The cavities of the mold are filled. (C) Particle harvesting: a filled mold is brought into contact with a high energy film or excipient layer (yellow) and passed through the heated nip without splitting. After cooling the mold is removed to reveal an array of particles on the high energy film or excipient layer. (D) PLGA microparticulate cubes with ridges and (E) PLGA microparticles with center fenestrations. Scale bars: (D) $3 \mu \mathrm{m}$, and (E) $20 \mu \mathrm{m}$ (Reprinted with permission from E. M. Enlow et al., Nano Lett. 11 (2011) 808-813. Copyright 2011 American Chemical Society). 


\subsection{CONCLUSIONS}

Most commercially available PLGA microsphere products can be classified as isotropic microspheres. Relevant studies used to focus on how to control their critical quality attributes such as particle size distribution, morphology, porosity, drug release pattern, and residual solvent content. This chapter summarizes interesting classes of anisotropic or next-generation PLGA microparticulate systems that are distinguished from commercially available PLGA microsphere products. It also provides various microfabrication techniques that allow the manufacturing of such anisotropic or geometryspecific PLGA microparticles. The ability to prepare tailor-made microparticle geometry is highly valuable as its shape and size have profound impacts on biological processes. Exploration of the use of anisotropic or geometry-specific microparticles in pharmaceutical applications has revealed their superiority compared to conventional monolithic microspheres. The novel PLGA microparticles with multifunctional architecture dealt in this chapter are likely to draw increasing attention for their wide applications in drug delivery, imaging, diagnostics, theranostics, sensors, photonics, and multi-hybrid composites.

\section{ACKNOWLEDGEMENTS}

This study was supported by the Korea SGER Program through the National Research Foundation of Korea (NRF) funded by the Ministry of Education, Science and Technology (NRF-2014R1A1A2A16054899).

\section{REFERENCES}

1. M. Okubo, A. Yamaguchi, T. Fujiwara. Colloid. Polym. Sci. 277 (1999) 1005-1008.

2. N. Konishi, T. Fujibayashi, T. Tanaka, H. Minami, M. Okubo. Polym. J. 42 (2010) 66-71.

3. Y.K. Takahara, S. Ikeda, S. Ishino, K. Tachi, K. Ikeue, T. Sakata, T. Hasegawa, H. Mori, M. Matsumura, B. Ohtani. J. Am. Chem. Soc. 127 (2005) 6271-6275.

4. S.H. Kim, C.J. Heo, S.Y. Lee, G.R. Yi, S.M. Yang. Chem. Mater. 19 (2007) 4751-4760.

5. $\quad$ M.R. Kim, S. Lee, J.K. Park, K.Y. Cho. Chem. Commun. 46 (2010) 7433-7435.

6. M.J. Schaefer, J. Singh. Biomaterials 23 (2002) 3465-3471.

7. F. Kang, J. Singh. AAPS PharmSciTech 2 (2001) article 30.

8. F. Mohamed, C.F. van der Walle. Int. J. Pharm. 311 (2006) 97-107.

9. M.R. Kim, Y.T. Lim, K.Y. Cho. Macromol. Rapid. Commun. 34 (2013) 406-410.

10. N. Rasheed, A.A. Khorasani, J. Cebral, F. Mut, R. Lohner, C. Salvador-Morales. Langmuir 31 (2015) 6639-6648.

11. B. Mandal, H. Bhattacharjee, N. Mittal, H. Sah, P. Balabathula, L.A. Thoma, G.C. Wood. Nanomedicine 9 (2013) 474-491. 
12. C. Salvador-Morales, B. Brahmbhatt, V. Márquez-Miranda, J. Araya-Duran, F. Gonzalez-Nio, C. Vilos, J. Cebral, F. Mut, R. Lohner, B. Leong, G. Sudaresan, J. Zweit. Langmuir 32 (2016) 7929-7942.

13. I.G. Loscertales, A. Barrero, I. Guerrero, R. Cortijo, M. Marquez, A.M. GananCalvo. Science 295 (2002) 1695-1698.

14. M. Enayati, M.W. Chang, F. Bragman, M. Edirisinghe, E. Stride. Colloids Surf. A Physicochem. Eng. Aspects 382 (2011) 154-164.

15. Y.H. Lee, F. Mei, M.Y. Bai, S. Zhao, D.R. Chen. J. Control. Release 145 (2010) 58-65.

16. J. Xie, W.J. Ng, L.Y. Lee, C.H. Wang. J. Colloid. Interface Sci. 317 (2008) 469-476.

17. S. Yuan, F. Lei, Z. Liu, Q. Tong, T. Si, R. X. Xu. PLosOne 10 (2015) e0132609.

18. H. Nie, Z. Dong, D.Y. Arifin, Y. Hu, C.H. Wang. J. Biomed. Mater. Res. A 95 (2010) 709-716.

19. M. Zamani, M.P. Prabhakaran, E.S. Tian, S. Ramakrishna. Int. J. Pharm. 473 (2014) 134-143.

20. Y. Namiki, T. Ueyama, T. Yoshida, R. Watanabe, S. Koido, T. Namiki. Sci. Rep. 4 (2014) article number: 6294.

21. C. Takai, T. Hotta, S. Shiozaki, Y. Boonsongrit, H. Abe. Chem. Commun. 37 (2009) 5533-5535.

22. L.C. Glangchai, M. Caldorera-Moore, L. Shi, K. Roy. J. Control. Release 125 (2008) 263-272.

23. J.M. DeSimone, S.J. Mecham, C.L. Farrell. ACS Cent. Sci. 2 (2016) 588-597.

24. J. Guan, N. Ferrell, L.J. Lee, D.J. Hansford. Biomaterials 27 (2006) 4034-4041.

25. G. Acharya, C.S. Shin, M. McDermott, H. Mishra, H. Park, I.C. Kwon, K. Park. J. Control. Release 141 (2010) 314-319.

26. G. Acharya, C.S. Shin, K. Vedantham, M. McDermott, T. Rish, K. Hansen, Y. Fu, K. Park. J. Control. Release 146 (2010) 201-206.

27. Y. Lu, M. Sturek, K. Park. Int. J. Pharm. 461 (2014) 258-269.

28. N. Malavia, L. Reddy, I. Szinai, N. Betty, J. Pi, J. Kanagaraj, A. Simonian, R. Jennings, G. Stoller. Invest. Ophthalmol. Vis. Sci. 56 (2015) 1296.

29. J.P. Rolland, B.W. Maynor, L. E. Euliss, A.E. Exner, G.M. Denison, J.M. DeSimone. J. Am. Chem. Soc. 127 (2005) 10096-10100.

30. E.M. Enlow, J.C. Luft, M.E. Napier, J.M. DeSimone. Nano Lett. 11 (2011) 808-813.

31. Y. Geng, P. Dalhaimer, S.S. Cai, R. Tsai, M. Tewari, T. Minko, D.E. Discher. Nat. Nanotechnol. 2 (2007) 249-255.

32. J.A. Champion, S. Mitragotri. Proc. Natl. Acad. Sci. USA 103 (2006) 4930-4934.

33. S. Muro, C. Garnacho, J.A. Champion, J. Leferovich, C. Gajewski, E.H. Schuchman, S. Mitragotri, V.R. Muzykantov. Mol. Ther. 16 (2008) 1450-1458.

34. J.H. Park, G von Maltzahn, L.L. Zhang, M.P. Schwartz, E. Ruoslahti, S.N. Bhatia, M.J. Sailor. Adv. Mater. 20 (2008) 1630-1635.

35. J.A. Champion, S. Mitragotri. Pharm. Res. 26 (2009) 244-249.

36. P. Decuzzi, R. Pasqualini, W. Arap, M. Ferrari. Pharm. Res. 26 (2009) 235-243.

37. S.E.A. Gratton, P.A. Ropp, P.D. Pohlhaus, J.C. Luft, V.J. Madden, M.E. Napier, J.M. DeSimone. Proc. Natl. Acad. Sci. USA 105 (2008) 11613-11618.

38. J.L. Perry, K.P. Herlihy, M.E. Napier, J.M. DeSimone. Acc. Chem. Res. 44 (2011) 990-998.

39. J.W. Yoo, N. Doshi, S. Mitragotri. Macromol. Rapid. Commun. 31 (2010) 142-148. 